\title{
Integrating risk preferences in forest harvest scheduling
}

\author{
Kyle Eyvindson $^{1} \cdot$ Annika Kangas $^{2}$
}

Received: 7 May 2015 / Accepted: 13 August 2015 /Published online: 11 September 2015

(C) INRA and Springer-Verlag France 2015

\begin{abstract}
- Key message Through a stochastic programming framework, risk preferences can be included in forest planning. The value of utilizing stochastic programming is always positive; however, the value depends on the information quality and risk preferences of the decision maker.

- Context Harvest scheduling requires decisions be taken based on imperfect information and assumptions regarding the future state of the forest and markets.

- Aims The aim of this study is to incorporate elements of risk management into forest management, so that the decision maker can understand the risks associated with utilizing the imperfect data.

- Methods Incorporation of uncertainty is done through stochastic programming. This allows for the decision maker's attitude towards risk to be incorporated into the development of a solution. By means of a simple even-flow problem formulation, a method of using stochastic programming to incorporate explicit trade-off between objective function value and risk of not meeting the constraints has been developed.
\end{abstract}

\section{Handling Editor: Barry Alan Gardiner}

Contribution of the co-authors Eyvindson: writing paper, data analysis, and method development.

Kangas: editing paper, method development, and supervision.

Kyle Eyvindson

kyle.eyvindson@helsinki.fi

Annika Kangas

Annika.kangas@luke.fi

1 Department of Forest Sciences, University of Helsinki, P.O. Box 27, FI-00014 Helsinki, Finland

2 Economics and Society, Natural Resources Institute Finland (Luke), P.O. Box 68, FI-80101 Joensuu, Finland
- Results The different models highlight the importance of including uncertainty in management of forest resources. In general, as the decision maker becomes more risk averse, the incorporation of uncertainty into the model becomes more important.

- Conclusions The use of stochastic programming allows for additional information to be included in the formulation, and this allows for the decision maker to account for downside risk.

Keywords Stochastic programming $\cdot$ Multiple criteria decision-making $\cdot$ Risk $\cdot$ Value of Information $\cdot$ Even-flow forestry

\section{Introduction}

Developing harvest scheduling plans (or more generally forest management plans) requires the use of imperfect information about the state of the forest and its future development, the preferences of forest owner, and the market. Information about the distribution of errors for most forest values can be statistically estimated (Gregoire and Valentine 2008). Uncertainty about timber price development or climate change development cannot be statistically estimated but could rely on expert opinions to evaluate the uncertainties (e.g., Leskinen and Kangas 2001). However, this information about the error is rarely used when developing a management plan (DiazBalteiro and Romero 2008). Typically, the planning starts with the assumption that both the inventory data and forecasting tools used accurately describe both the current and future state of the forest. Plans are then created which perform optimally, subject to strict constraints, for the very specific case where the current and future state of the forest matches the estimation/prediction. By choosing to ignore the estimates of error in 
the information, the planner removes the ability to actively manage the risks of meeting either the strict constraints or the optimization of the objective function.

In the pioneering book by Knight (1921), a differentiation between risk and uncertainty has been made where risk has a known probability, and uncertainty has an unknown probability. For many situations, this definite distinction is obscured, for instance while we do not know the exact probability of a piece of information, we have reasonable estimates of the probabilities. So while we may be operating under a case of uncertainty, by using reasonable estimates of the probabilities, we can analyze the situation by using methods which operate under risk. Management of risks falls into three different categories in forests: resources (relating to the production and development), markets (price uncertainty, both costs and revenues), and preferences (decision makers and policy makers). The focus of this paper will be on the resource risk of imperfect inventory measurements; however, the theoretical framework could integrate the risk from both market and preferential sources.

While there has been substantial research conducted into analyzing the errors of forest inventory methods (e.g., Mäkinen et al. 2010), little research has been done to manage these risks according to the risk preferences of the decision maker(s) (DM). The focus of the literature regarding uncertainty in forest management has focused on ensuring that the flow constraints are met under uncertainty according to some given probability. Hof and Pickens (1991) used a chanceconstrained and chance-maximizing approach to produce plans which minimized the probability of not meeting demand for three harvest products. Palma and Nelson (2009) used robust optimization to protect against the uncertainty of the yield and demand of timber, while maximizing net present value. These methods allowed risk-averse DMs to ensure with a high level of probability that the constraints remain feasible (Bertsimas and Sim 2004). However, this feasibility may be overemphasized in many of the cases (see e.g., Mulvey et al. 1995). For instance, in the traditional even-flow problem, requiring exactly even-flow is usually not that important, and in many cases, the flow is required to stay, say, within $3 \%$ of the first period flow (e.g., Duvemo et al. 2014). This formulation might make better sense than exact even-flow in deterministic problem formulation, but in the robust programming context, both formulations may be too expensive for the DM with respect to the objective function value.

Other researchers (Eid 2000; Islam et al. 2010) have focused on evaluating the impact inventory errors have on the achievement of goals (i.e., net present value) at a stand-wise level. This approach incorporates error into the decisionmaking process; however, this does not allow the DM to provide preferences on how the risk should be managed. Through a stochastic programming formulation, Kangas et al. (2014) have produced a method which can be used to indicate where updating forest inventory data is valuable, which could be modified to incorporate the risk preferences of an individual DM. Additionally, through the use of stochastic goal programming, Eyvindson and Kangas (2014) have created a method to develop management plans which implicitly accounts for the risk preferences of the DM through the problem formulation through minimizing the risk of not achieving the targets set.

Explicit accounting of risk measures in forest management planning is something which has not received much attention. Piazza and Pagnoncelli (2014) have developed a model which provides optimal policies to maximize net present value considering price uncertainty. If additional constraints are included, for instance, an even-flow of income through the planning horizon, the risk of not meeting these constraints should be addressed. The novelty of the approach described here is that risk is explicitly accounted for, and the decision maker can determine the trade-off between the objective function value and ensuring the even-flow policy.

A significant amount of literature on managing risk in a forestry setting relates to managing the costs caused by natural hazards (Hanewinkel et al. 2011). Accounting for the impact of natural hazards is important and can significantly influence how a forested area will be managed (Savage et al. 2011). Risk preferences are specific to an individual or group and depend upon which aspects of forest management are important to the $\mathrm{DM}$ and how these aspects relate to each other. For instance, if a DM wishes to maximize (minimize) a single forest variable where the risk cannot be managed based on the treatment options selected, then understanding the DM's risk preferences are not required. If the risks to the stands depend on the treatment options selected (e.g., risk of fire or wind-throw), then the management focus is a trade-off between maximizing the objective function and minimizing the risk of natural hazards. Likewise, when the DM has additional constraints, such as a requirement for an even flow of income, the management focus is a trade-off between the objective value and risk of not meeting those constraints.

The management of forest resources is risky, and DMs should have the opportunity to have an impact on those aspects of risk which are manageable. To do this, we propose using stochastic programming (Birge and Louveaux 2011; King and Wallace 2012) as a method to incorporate the inherent risks of making decisions with imperfect information. The approach described in this paper reflects the robust optimization method described by Mulvey et al. (1995), where they balance the objective value function with the feasibility of the problem under a set of scenarios. It can be interpreted as an inherently two-stage stochastic programming approach with the second stage described with penalties (Birge and Louveaux 2011). As the mitigation of risk is a trade-off between two or more criteria, we propose an approach to first evaluate the riskneutral situation and then use weights to balance between the objectives. Through value of information calculations, we 
highlight how the solution improves when incorporating both the risk preferences and uncertainty estimates. This is demonstrated through a general formulation which allows for an even-flow of income while ensuring an end productive value, so that the expected future harvesting opportunities are at least the same as the initial state (Pukkala 2005).

\section{Methods}

A general formulation is proposed which maximizes the first period income, while minimizing the negative deviations from either the first period income (denoted by $\mathbf{A}$ ), or the previous period income (denoted by $\mathbf{B}$ ) and the negative deviations from the end productive value constraint. The productive value is calculated as the discounted value of all future incomes in the forest, based on the species specific models of Pukkala (2005), which uses a set of variables as predictors (basal area, mean diameter at breast height, discount rate, site variables, and timber price). These formulations represent slightly different interpretations of even-flow forest management. While market risk is not included in this analysis, as constant prices were used, income and productive value were used to aggregate the value of timber as DMs may find the use of incomes to be a more beneficial tool for making comparisons between alternative plans.

The even-flow problem formulation explicitly states the preference for income throughout the planning horizon. The DM wishes to obtain as large of an initial income as possible, while ensuring future periods will be able to provide the same level of income. Three different models will be presented: the expected value (EV) model, which uses the specific case where the original inventory measurement errors are ignored; the simple recourse problem (RP) model, which incorporates the estimated errors through a generation of a large number of scenarios; and the wait-and-see (WS) model, which assumes that perfect information can be obtained, and the correct decision taken for each scenario.

The proposed EV model solves the problem using the single scenario which ignores the measurement errors of the original inventory:

$\max E V=\sum_{j=1}^{J} \sum_{k=1}^{K_{j}} \mathbb{E}\left(c_{j k 1}\right) x_{j k}-\lambda\left(\sum_{t=1}^{T}\left(w_{t}^{-} d_{t}^{-}\right)+w_{E}^{-} d_{E}^{-}\right)$

subject to:

$$
\begin{aligned}
\sum_{j=1}^{J} \sum_{k=1}^{K_{j}} \mathbb{E}\left(c_{j k t}\right) x_{j k}-\sum_{j=1}^{J} \sum_{k=1}^{K_{j}} \mathbb{E}\left(c_{j k 1}\right) x_{j k} \\
-d_{t}^{+}+d_{t}^{-}=0, \forall t=2, \ldots, T
\end{aligned}
$$

$$
\begin{aligned}
& \sum_{j=1}^{J} \sum_{k=1}^{K_{j}} \mathbb{E}\left(c_{j k t}\right) x_{j k}-\sum_{j=1}^{J} \sum_{k=1}^{K_{j}} \mathbb{E}\left(c_{j k t-1}\right) x_{j k} \\
& -d_{t}^{+}+d_{t}^{-}=0, \forall t=2, \ldots, T \\
& \sum_{j=1}^{J} \sum_{k=1}^{K_{j}} \mathbb{E}\left(P V_{j k T}\right) x_{j k}-d_{E}^{+}+d_{E}^{-}=\sum_{j=1}^{J} \mathbb{E}\left(P V_{j 0}\right) \\
& \sum_{k=1}^{K_{j}} x_{j k}=1, \forall j=1, \ldots, J \\
& x_{j k} \in[0,1] \\
& d_{t}^{+}, d_{t}^{-} \geq 0, \forall t=2, \ldots, T \\
& d_{E}^{+}, d_{E}^{-} \geq 0
\end{aligned}
$$

where $\mathbb{E}$ is the expected value for the variable in question, $x_{j k}$ is the decision variable used to decide the proportion of stand $j$ being managed using schedule $k$, where a schedule is a set of harvesting (e.g., thinning or final felling) or management actions (e.g., planting, fertilizing or pre-commercial thinnings) taken during the planning periods. $\mathbb{E}\left(c_{j k t}\right)$ is the expected value of incomes (calculated as volume of timber harvested multiplied by price per cubic meter minus silvicultural costs) in stand $j$, schedule $k$, and period $t$ and $d_{t}^{-}\left(d_{t}^{+}\right)$are decision variables for determining the negative (positive) deviation of income in period $t$ compared to the first period income (A) or previous period income $(\mathbf{B}), \mathbb{E}\left(P V_{j k T}\right)$ is the expected productive value of the stand $j$ in schedule $k$ at the end of the planning horizon and $\mathbb{E}\left(P V_{j 0}\right)$ is the expected productive value at the beginning of the planning horizon, $w_{t}^{-}\left(w_{t}^{+}\right)$and $w_{E}^{-}\left(w_{E}^{+}\right)$are parameters set by the $\mathrm{DM} /$ forest planner indicating the penalties associated with negative (positive) deviations, and $\lambda$ is a risk coefficient which is dependent on the DM's risk aversion preferences; it balances the deviations of the even-flow problem and the maximization of the first period income. In a similar way as Noyan (2012), $\lambda$ acts as a tradeoff coefficient, representing the exchange rate for the mean cost of risk. So as the DM's appetite for risk decreases, $\lambda$ should increase. $d_{E}^{-}\left(d_{E}^{+}\right)$is the decision variable for determining the negative (positive) deviation from the first to the last period for the productive value of the forest, $T$ is the number of periods (6), and $J$ is the number of stands and $K_{j}$ the number of treatment schedules for stand $j$. To ease the readability of the equations, Table 1 provides a list of the variables used in this paper. Constraint $2 \mathbf{A}$ corresponds to the case where the evenflow is compared to the first period, while constraint $2 \mathbf{B}$ corresponds to the case where the even-flow is compared to the previous period.

This formulation ignores the uncertainty inherent in the inventory data and thus cannot manage the risk involved in managing the negative deviations. As the constraints are soft, even when introducing uncertainty, the solution obtained will be feasible. 
Table 1 A list of the notation used throughout the paper

\begin{tabular}{|c|c|}
\hline Symbol & Definition \\
\hline \multicolumn{2}{|c|}{ Variables, measured in $€$ or $€ /$ period } \\
\hline$c_{j k t}$ & Income, for stand $j$, schedule $k$, at period $t$ \\
\hline$c_{j k i t}$ & Income, for stand $j$, schedule $k$, for scenario $i$, at period $t$ \\
\hline $\mathbb{E}$ & Expectation value \\
\hline$d_{t}^{-}, d_{t}^{+}$ & Negative (positive) deviations during time period $t$ \\
\hline$d_{E}^{-}, d_{E}^{+}$ & Negative (positive) deviations for the difference between initial and end productive value \\
\hline$d_{i t}^{-}, d_{i t}^{+}$ & Negative (positive) deviations for scenario $i$ during time period $t$ \\
\hline$d_{i E}^{-}, d_{i E}^{+}$ & Negative (positive) deviations the difference between initial and end productive value for scenario $i$ \\
\hline $\bar{d}_{i t}$ & Observed negative deviations for scenario $i$ during time period $t$ \\
\hline $\bar{d}_{i E}$ & Observed negative deviations the difference between initial and end productive value for scenario $i$ \\
\hline$p_{i}$ & Probability of scenario $i$ occurring \\
\hline$P V_{j k T}$ & Productive value for stand $j$, schedule $k$, at time $T$ \\
\hline$P V_{j 0}$ & Productive value for stand $j$, at the start of the planning horizon \\
\hline$P V_{j k i T}$ & Productive value for stand $j$, schedule $k$, for scenario $i$, at time $T$ \\
\hline$P V_{j 0 i}$ & Productive value for stand $j$, at the start of the planning horizon for scenario $i$ \\
\hline$z_{i}$ & Optimal objective function value for scenario $i$ \\
\hline \multicolumn{2}{|c|}{ Variables, measured in \% } \\
\hline$x_{j k}$ & Proportion of stand $j$ managed according to schedule $k$ \\
\hline$x_{j k i}$ & Proportion of stand $j$ managed according to schedule $k$ for scenario $i$ \\
\hline $\begin{array}{l}\bar{x}_{j k} \\
\text { Paramete }\end{array}$ & Optimal solution for the EV problem (Eq. (1)) \\
\hline$w_{t}^{-}, w_{t}^{+}$ & Weight assigned to negative (positive) deviations during time period $t$ \\
\hline$w_{E}^{-}, w_{E}^{+}$ & Weight assigned to negative (positive) deviations for the difference between initial and end productive value \\
\hline$\lambda$ & Risk coefficient \\
\hline
\end{tabular}

To incorporate uncertainty into the problems, we propose the following RP formulation. This problem is solved for a large number of scenarios simultaneously, generating a single, implementable decision for all scenarios:

$\max R P=\sum_{i=1}^{I} \sum_{j=1}^{J} \sum_{k=1}^{K_{j}} p_{i} c_{j k i 1} x_{j k}-\lambda \sum_{i=1}^{I} p_{i}\left(\sum_{t=1}^{T}\left(w_{t}^{-} d_{i t}^{-}\right)+w_{E}^{-} d_{i E}^{-}\right)$

Subject to:

$$
\begin{aligned}
& \sum_{j=1}^{J} \sum_{k=1}^{K_{j}} c_{j k i t} x_{j k}-\sum_{j=1}^{J} \sum_{k=1}^{K_{j}} c_{j k i 1} x_{j k}-d_{i t}^{+}+d_{i t}^{-}=0, \forall i \\
& \quad=1, \ldots, I, t=2, \ldots, T
\end{aligned}
$$

$$
\begin{aligned}
& \sum_{j=1}^{J} \sum_{k=1}^{K_{j}} c_{j k i t} x_{j k}-\sum_{j=1}^{J} \sum_{k=1}^{K_{j}} c_{j k i t-1} x_{j k}-d_{i t}^{+}+d_{i t}=0, \forall i \\
& \quad=1, \ldots, I, t=2, \ldots, T \\
& \sum_{j=1}^{J} \sum_{k=1}^{K_{j}} P V_{j k i T} x_{j k}-d_{i E}^{+}+d_{i E}^{-}=\sum_{j=1}^{J} P V_{j 0 i}, \forall i=1, \ldots, I \\
& d_{i t}^{+}, d_{i t}^{-} \geq 0, \forall i=1, \ldots, I, t=2, \ldots, T \\
& d_{i E}^{+}, d_{i E}^{-} \geq 0, \forall i=1, \ldots, I
\end{aligned}
$$

and subject to Eqs. (4) and (5)

where subscript $i$ indicates that the variable is associated with scenario $i$, and $I$ refers to the total number of scenarios under consideration. $c_{j k i t}$ is the value of net incomes in stand $j$, schedule $k$, scenario $i$, and period $t, P V_{j k i T}$ is the productive value of the stand $j$ in schedule $k$ for scenario $i$ at the end of the 
planning horizon and $P V_{j i}$ is the productive value at the beginning of the planning horizon for scenario $i$, and $p_{i}$ refers to the probability of scenario $i$ occurring.

The uncertainty is incorporated through a large set of scenarios, a deterministic equivalent of the stochastic problem. This allows the problem to be computationally tractable. The size of the RP problem is $I$ times the size of the EV problem. Determining the specific number of scenarios depends on how the uncertainty is used in the problem formulation (King and Wallace 2012), and the approximation must be appropriate for the stochastic model which is to be used. In this formulation, the penalties act in a similar fashion as in the EV formulation; however, in this case, they reflect the importance of the expected negative deviations across all scenarios rather than exact negative deviations for one scenario provided by the EV formulation.

To understand how much improvement can occur if we were to obtain perfect information, we propose following the WS formulation. This problem solves each scenario independently, creating an optimal decision for each scenario:

$\mathrm{WS}=\sum_{i=1}^{I} p_{i} z_{i}, \forall i=1, \ldots, I$

For all $i=1, \ldots, I$ (Eqs. (14), (15A) or (15B), (16), (17), and (18))

$\max z_{i}=\sum_{j=1}^{J} \sum_{k=1}^{K_{j}} c_{j k i 1} x_{j k i}-\lambda\left(\sum_{t=1}^{T}\left(w_{t}^{-} d_{i t}^{-}\right)+w_{E}^{-} d_{i E}^{-}\right)$

Subject to

$\sum_{j=1}^{J} \sum_{k=1}^{K_{j}} c_{j k i t} x_{j k i}-\sum_{j=1}^{J} \sum_{k=1}^{K_{j}} c_{j k i 1} x_{j k i}-d_{i t}^{+}+d_{i t}^{-}=0, \forall t=2, \ldots, T$

$\sum_{j=1}^{J} \sum_{k=1}^{K_{j}} c_{j k i t} x_{j k i}-\sum_{j=1}^{J} \sum_{k=1}^{K_{j}} c_{j k i t-1} x_{j k i}-d_{i t}^{+}+d_{i t}^{-}=0, \forall t=2, \ldots, T$

$\sum_{j=1}^{J} \sum_{k=1}^{K_{j}} P V_{j k i T} x_{j k i}-d_{i E}^{+}+d_{i E}=\sum_{j=1}^{J} P V_{j 0 i}$

$\sum_{k=1}^{K_{j}} x_{j k i}=1, \forall j=1, \ldots, J$

$x_{j k i} \in[0,1]$

and subject to Eqs. (11) and (12).

This formulation calculates the expected value of making perfect decisions for all scenarios and indicates how valuable perfect information can be.
In order to make the results of the EV problem comparable to the WS and RP problems, we need to calculate the expected result of the EV problem (EEV). This problem just integrates the decision determined by the EV problem and generates a comparable solution to the problems which uses the entire set of scenarios.

For the EEV solution, let us denote $\bar{x}_{j k}$ as the optimal solution to [1]

$E E V=\sum_{i=1}^{I} \sum_{j=1}^{J} \sum_{k=1}^{K_{j}} p_{i} c_{j k i 1} \bar{x}_{j k}-\lambda \sum_{i=1}^{I} p_{i}\left(\sum_{t=1}^{T}\left(w_{t}^{-} \bar{d}_{i t}^{-}\right)+w_{E}^{-} \bar{d}_{i \bar{E}}\right)$

$\bar{d}_{i t}^{-}\left\{\begin{array}{c}0 \text { if } \sum_{j=1}^{J} \sum_{k=1}^{K_{j}} c_{j k i 1} \bar{x}_{j k}<\sum_{j=1}^{J} \sum_{k=1}^{K_{j}} c_{j k i} \bar{x}_{j k}, i=1, \ldots, I, t=2, \ldots, T \\ \sum_{j=1}^{J} \sum_{k=1}^{K_{j}} c_{j k i 1} \bar{x}_{j k}-\sum_{j=1}^{J} \sum_{k=1}^{K_{j}} c_{j k i} \bar{x}_{j k}, i=1, \ldots, I, t=2, \ldots, T, \text { otherwise }\end{array}\right.$

$\bar{d}_{i t}^{-}\left\{\begin{array}{c}0 \text { if } \sum_{j=1}^{J} \sum_{k=1}^{K_{j}} c_{j k i t-1} \bar{x}_{j k}<\sum_{j=1}^{J} \sum_{k=1}^{K_{j}} c_{j k i} \bar{x}_{j k}, i=1, \ldots, I, t=2, \ldots, T \\ \sum_{j=1}^{J} \sum_{k=1}^{K_{j}} c_{j k i t-1} \bar{x}_{j k}-\sum_{j=1}^{J} \sum_{k=1}^{K_{j}} c_{j k i t} \bar{x}_{j k}, i=1, \ldots, I, t=2, \ldots, T, \text { otherwise }\end{array}\right.$

$\bar{d}_{i \bar{E}}\left\{\begin{array}{c}0 \text { if } \sum_{j=1}^{J} P V_{j 0 i}<\sum_{j=1}^{J} \sum_{k=1}^{K_{j}} P V_{j k i} \bar{x}_{j k}, i=1, \ldots, I \\ \sum_{j=1}^{J} P V_{j 0 i}-\sum_{j=1}^{J} \sum_{k=1}^{K_{j}} P V_{j k i} \bar{x}_{j k}, i=1, \ldots, I, \text { otherwise }\end{array}\right.$

and subject to Eqs. (11) and (12).

where $\bar{d}_{i t}^{-}$are the observed negative deviations (in the EV problem, they are results of a decision but not decision variables as in RP problem) of the EV decision under scenario $i$ in period $t$ and $\bar{d}_{i \bar{E}}$ are the observed negative deviations from the first to the last period for the productive value of the forests for scenario $i$.

From these formulations, it is possible to calculate the value of information (VOI). The VOI is calculated as the possible improvement of the solution by obtaining perfect information or by including the uncertainty in the problem (Chapter 4 of Birge and Louveaux 2011). The expected value of perfect information (EVPI) measures the costs a DM may encounter for not obtaining perfect information. The value of the stochastic solution (VSS) reflects the cost of making a decision without considering the uncertainty, for a maximization problem the calculations are:

$E V P I=W S-R P$

$V S S=R P-E E V$ 
In order to analyze how the risk preferences influence both the solutions and the VOI, relevant weights for the penalties need to be determined. While the weights should be determined by the DM, a suitable surrogate is the shadow prices from the constraints of the EV problem (Eqs. (2A) or (2B) and (3)) when the weights are initially set at an arbitrary high value. This is justified as this EV problem describes an underlying implicit utility model for the time preferences of income and the trade-off between incomes and the end value of the forests, which is applicable in the vicinity of the optimal solution (Lappi 1992). To highlight the impact of changing risk preferences regarding the amount of weighted negative deviations, $\lambda$ will be set at one and increased by increments of 0.05 until it reaches six. This reflects a shift from a nearly riskneutral preference to an increasingly risk-averse preference.

For the formulation presented here, when the penalties are interpreted as reflecting the time and flexibility preferences for negative deviations; $\lambda$ should not be assigned a value less than one. The primary justification is due to the general structure of the formulation, it is designed to minimize the downside losses dependent on the first or previous period income. As such, if the DM were a risk seeker, then the general problem formulation would need to be adjusted to better suit those preferences.

To find solutions to the problem formulations, the optimization software IBM ILOG CPLEX Optimization Studio V12.6 was used. For problems of this size, non-proprietary software (such as the LP-solve package available through R) is not adequate.

\section{Materials}

A small (47.3 ha) privately held forest holding in North Karelia, Finland, consisting of 41 stands is used as a demonstration example. According to a recent inventory, the holding was composed primarily of Scots pine (Pinus sylvestris L.), with a fairly even age-class distribution (Fig. 1). The prices for timber and the cost for silvicultural activities were based on the most recent historical average (Peltola 2014). The planning period was for a 30-year time horizon, with six 5-year periods and operations occurring at year 2 of each period. A discount rate of $2 \%$ was used in the model to evaluate the productive values of the stands.

For this case study the only stochastic feature under consideration was that of inventory errors. A Monte Carlo simulation was used to generate a set of 100 scenarios, so each scenario represents an equally probable initial inventory for the forest holding (i.e., $p_{i}=\frac{1}{100}$, for all $i$ in $I$ ). Measurement errors were introduced to both the dominant height and to the basal area. Both errors were assumed to be normally distributed, had no bias, and had a relative standard error of $20 \%$,
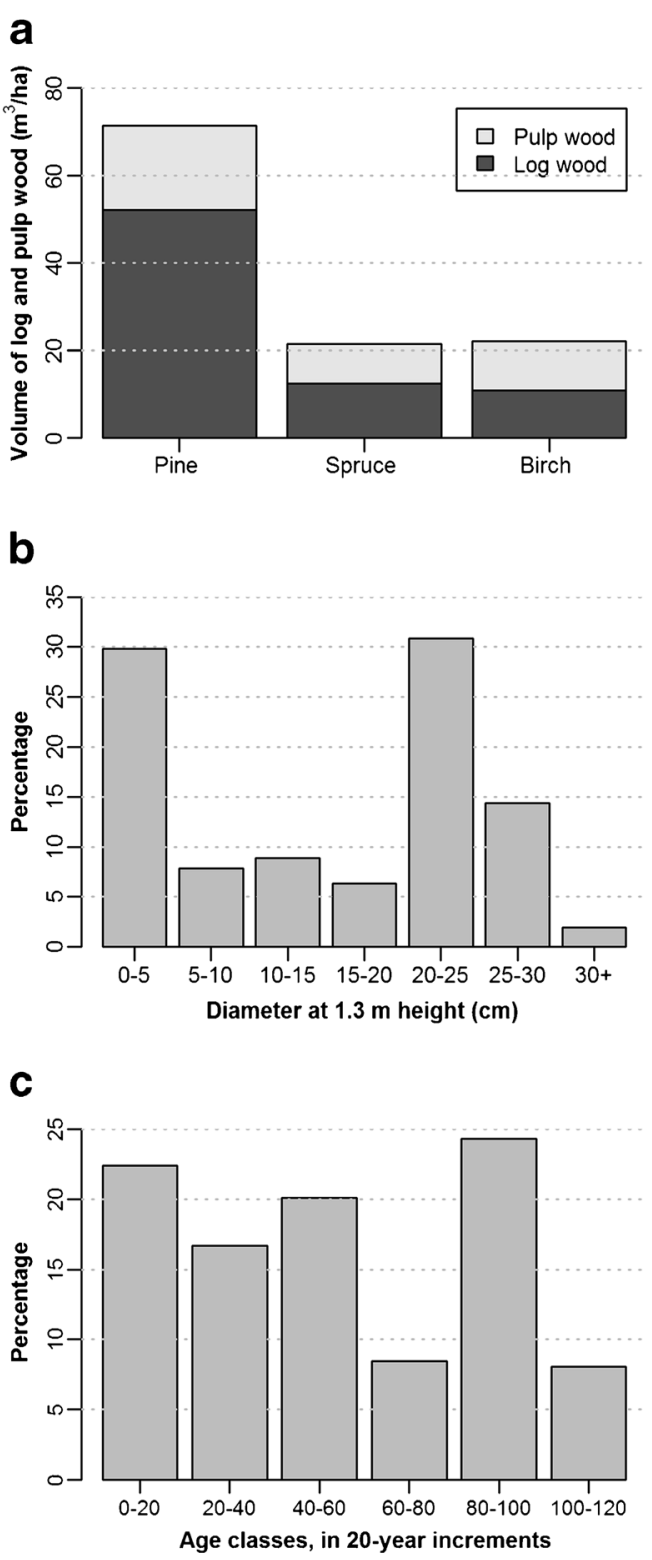

Fig. 1 a Volume of log and pulp wood in the holding at the beginning of the planning period, $\mathbf{b}$ diameter distribution at $1.3 \mathrm{~m}$ height in the holding at the beginning of the planning period, and $\mathbf{c}$ age-class distribution for the holding at the beginning of the planning period

which is a reflection of the state-of-the-art inventory methods (Næsset 2004). The measurements were considered uncorrelated, as recent studies have shown only a small variable correlation, varying from -0.22 to 0.11 (Haara and Korhonen 2004; Mäkinen et al. 2010).

\section{Results}

\subsection{The expected value (EV) problem}

For each EV problem, there are only two solutions. For the case when $\lambda>1$, the constraints are no longer soft and both $\mathbf{A}$ 
and $\mathbf{B}$ formulations have the same solution. The solution maximizes first period income and ensures an exact even-flow of income, with the productive value at the end equal to the productive value at the beginning. For the case when $\lambda=1$, the constraints soften, and the formulations produce unique solutions. For the case where uncertainty is ignored, formulation $\mathbf{A}$ allows for negative deviations only between periods 2 and 5 (Table 2), while formulation $\mathbf{B}$ allows for negative deviations only in period 5 . The same solutions are used in the EEV formulation. Neither formulation has managed the risk, but from the EEV solution, it is possible to calculate the distribution of observed incomes across the scenarios.

For the EV problem, adjusting $\lambda$ has no impact on the management of the negative deviations when considering uncertainty, as the model does not incorporate the error information. For the expected result of the EV solution (the EEV solution), increasing $\lambda$ has the impact of increasing the weighted sum of negative deviations calculated for the two different solutions across the set of scenarios.

\subsection{The simple recourse problem (RP)}

For the majority of iterations of $\lambda$ (over $99 \%$ ), both of the RP formulations generate a unique solution. This demonstrates that for a specific risk preference a unique solution will be provided. When $\lambda$ is small, a small change in $\lambda$ causes a dramatic change in the solution provided. Correspondingly, when $\lambda$ is large, a small change in $\lambda$ causes only a very small change in the solution provided. This highlights that the cost to implementing progressively risk-averse solutions is initially small, but the costs become greater. Both formulations $\mathbf{A}$ and B produce rather similar results. The key difference is that when $\lambda$ increases, the later period income is higher for formulation $\mathbf{B}$ than formulation $\mathbf{A}$. In the $\mathbf{A}$ formulation, the first period incomes decrease clearly as $\lambda$ increases. In $\mathbf{B}$ formulation, the reduction is less clear. The selection of which formulation is more appropriate is dependent on the risk preferences of the DM.

In order to manage risk, the DM can incur a cost in expected total income and PV. The total incomes and end productive value for most of the RP solutions are slightly less than the EEV solution. This loss is part of the trade-off of accounting for the risk. The risk aversion and its costs can be clearly seen from the worst case results. The more risk averse the DM (the greater the $\lambda$ ), the higher the worst-case incomes. The first period is an exception, however, as it is the objective. Then, when the DM is more risk averse, the cost is the decreasing objective function value.

\subsection{The wait-and-see problem}

The WS problem represents the special case when the uncertainty for each scenario becomes known, and the optimal selection of schedules is made for each scenario. When using the EV shadow prices as penalties, only a few unique solutions are generated. In a similar fashion as the EV problem, when $\lambda$ increases, the WS solution quickly shifts towards finding the solutions for each scenario so that the flow is exactly even, i.e., there are no negative deviations. This occurs for both formulations when $\lambda$ reaches 1.45 .

\subsection{Value of information}

The VOI measures the difference between the various formulations (EEV, RP, and WS) for a particular $\lambda$, with a constant set of weights. In this particular case, $\lambda$ represents the risk preference for obtaining even-flow. The value of the stochastic solution (VSS) measures the difference between EEV and RP, representing the value of incorporating uncertainty into the problem (Fig. 2). Both formulations demonstrate a similar trend in the VSS: an initial decrease, followed by a steady increase. Formulation $\mathbf{A}$ has a slight anomaly at the beginning of the VSS curve, moving from $\lambda=1$ to $\lambda=1.05$. This anomaly can be attributed to the fact that the EV shadow prices only estimate the risk-neutral weights for the penalties. The EVPI is the difference between the WS problem and the RP. It measures the expected value from obtaining perfect information before decisions are taken. For both formulations, the EVPI is rather similar; it starts rather low (around $0.5 \%$ ) and increases to about $9 \%$.

\section{Discussion}

When developing an optimization formulation to incorporate the DM's preferences, it is crucially important to model the preferences appropriately. To add value to the decisionmaking process, the solution provided must be an accurate reflection of the desires of the DM (Diaz-Balteiro et al. 2013). The method proposed in this paper provides for additional information to be integrated into the formulation, which can hopefully more accurately reflect the preferences elicited. This method may be rather technical for DMs or planners to understand; however, the tool could be made more accessible by utilizing easy to understand terms and avoiding the mathematical terms. The benefit of this approach is that uncertainty and risk can be included in the decision-making process using risk specific preferences.

The evaluation and modeling of a DMs preferences is of critical importance when developing a user specific management plan. Different DMs will have different priorities for their forests. Some forest owners may highly value berry picking or hunting opportunities and may be willing to delay harvesting opportunities to promote these activities. So, by accounting for risk, forest owners can provide preferences which specifically relate to risk. Future research could focus on 
प艹

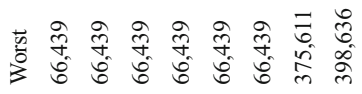

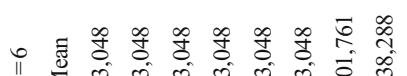

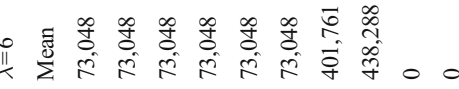

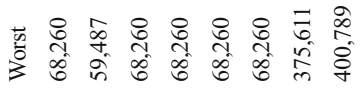

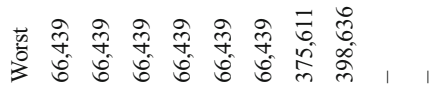

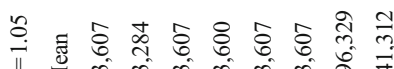

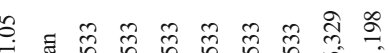

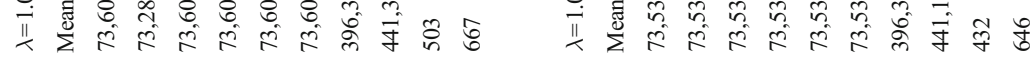

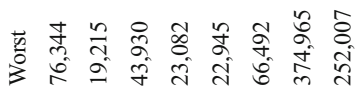

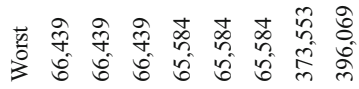

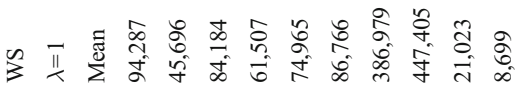

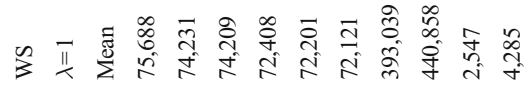

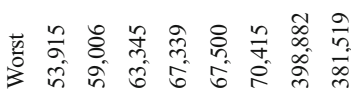

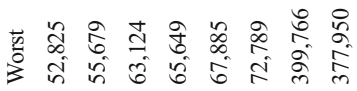

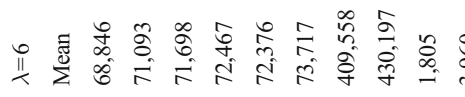

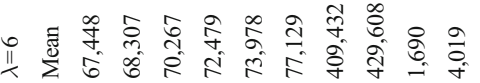

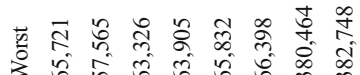

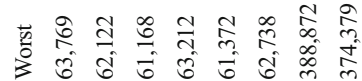

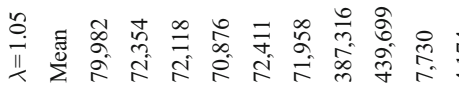

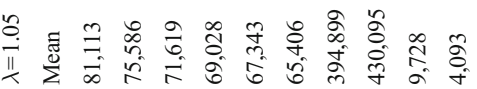

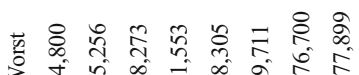

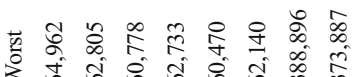

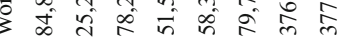

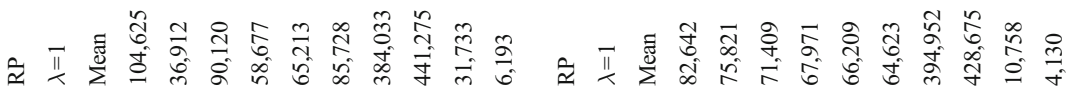

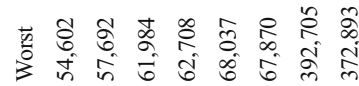

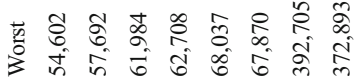

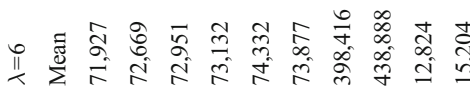

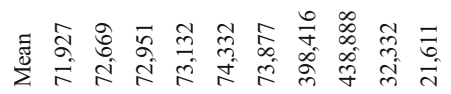

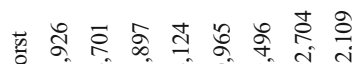

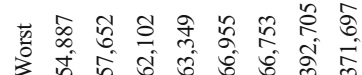

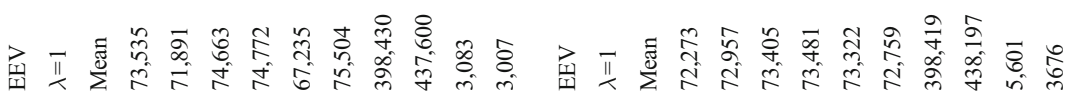

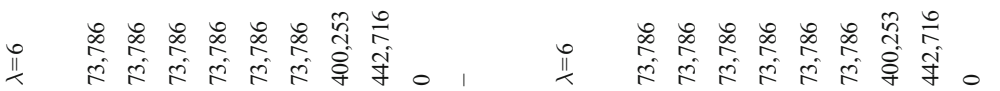

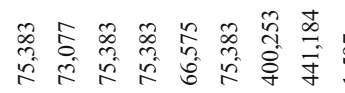

空, II

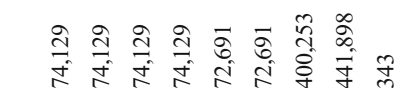

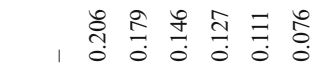

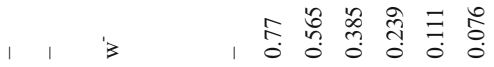

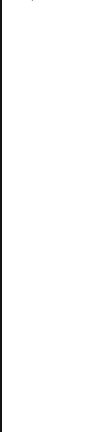
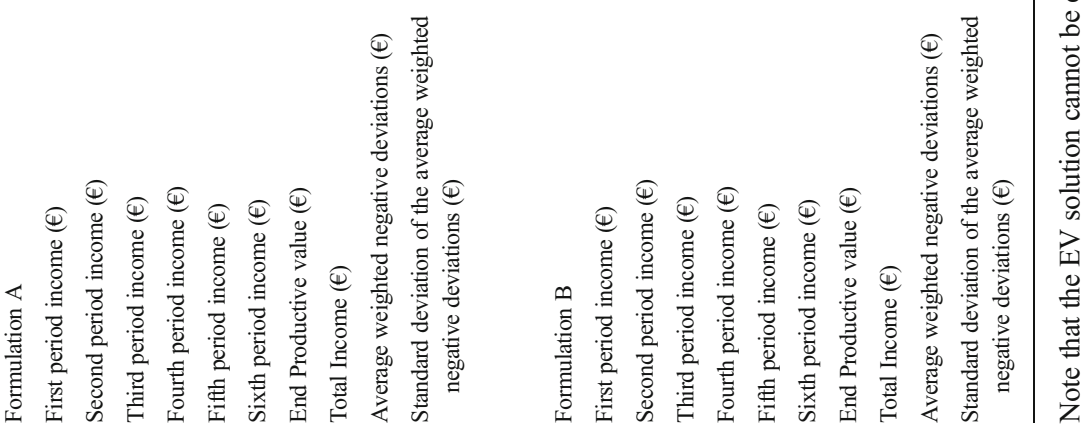
Value of Information

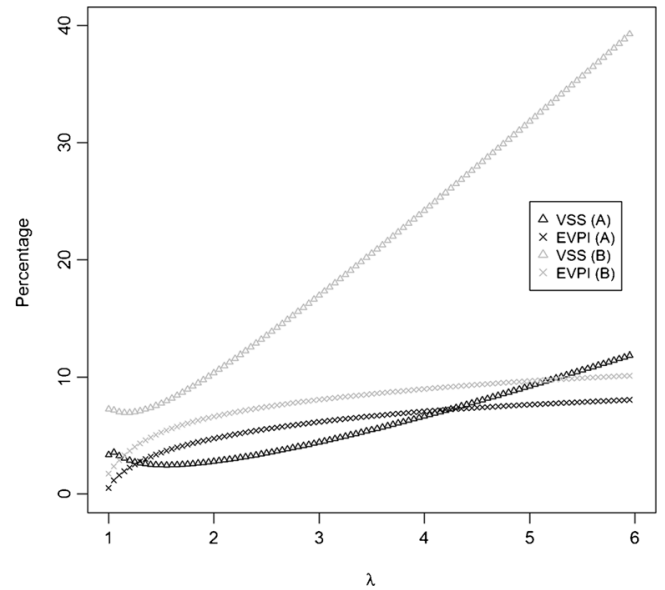

Fig. 2 The percentage change in the Value of Information as $\lambda$ increases from 1 to 6 for formulations $\mathbf{A}$ and $\mathbf{B}$

developing methods of collecting risk preferences in addition to traditional forest management preferences.

The problem presented in this paper is for a small holding with a moderate level of error in the initial inventory data. For cases where the absolute level of errors increase (a holding with more stands, or increased inventory error), both the VSS and EVPI will be larger. We have tested this by both changing the amount of error and by changing the size of the holding. Additionally, if additional sources of uncertainty are included (i.e., market uncertainty, or growth prediction uncertainty), the amount of variability in the scenario set will also increase. When the amount of variability increases (either through increased error or increased holding size) both the VSS and EVPI increase.

For the formulation proposed, the downside risk is minimized according to the risk preferences of the DM. For the case where the EV shadow prices were used as weight of the penalty, the selection of $\lambda$ reflects the importance of obtaining a solution which promotes an even-flow of income. The intent of using the EV shadow prices is to provide an estimation of how a risk-neutral DM would set weights reflecting the time preferences. The approximation of weights is seen through the results of the RP problem using formulation $\mathbf{A}$ and EV shadow prices as weights $(\lambda=1$, Table $2 \mathrm{a})$. With these weights, the problem does not assign enough importance to even-flow, and the objective function primarily maximizes first period income. However, with only a slight increase $(\lambda=1.05)$, the flow of income becomes much more even and is much more reasonably risk-neutral. As $\lambda$ is a constant, an assumption is made that the risk preferences of the individual are constant throughout the entire planning horizon. If the DM's risk preferences change, then an updated plan should be created.

The changes in VOI due to the shift from risk-neutral preferences to more risk-averse preferences are rather interesting (Fig. 2). For both formulations, the VSS is the lowest when $\lambda$ is slightly above 1 (1.55 for formulation $\mathbf{A}$, and 1.20 for formulation B). This indicates that the improvement caused by the shift from the EV formulation to the RP formulation is not as valuable for slightly risk-averse individuals as for both riskneutral and strongly risk-averse DM's. The reason for this is that the EV formulation produces only two solutions (where $\lambda=1$ and $\lambda>1$ ). The EV solution where $\lambda=1$ is most similar to the RP solution where $\lambda=1$, and the EV solution where $\lambda>1$ is most similar to the RP solution where $\lambda$ is slightly greater than 1. In this way, the $E V \lambda>1$ produces a solution which is more similar to the slightly risk-averse case than any of the other specific risk preference.

For both formulations, the EVPI is initially rather low, then increases quickly and levels off to increase at a steady rate. This shows that for a risk-neutral individual, there is very little value attributed to acquiring perfect information, while the value of more accurate information steadily increases according to how risk averse the DM is. In a way, the EVPI is related to the risk premium. The EVPI reflects the maximum amount a DM should be willing to pay to have a risk free alternative. While the EVPI for both formulations are rather similar (the difference ranges between 1 and $2 \%$ ), the VSS are substantially different. This primary difference in the VSS is due to the differences in the scale of the penalties attached to formulations. The sum of the penalties for formulations $\mathbf{A}$ and $\mathbf{B}$ are 0.85 and 2.15, respectively, which indicates that the VSS for formulation A should be about $40 \%$ of the VSS for formulation $\mathbf{B}$, which it is. This is due to the differences in the shadow prices between the formulations. For formulation $\mathbf{A}$, the shadow prices reflect the individual variable shadow price, whereas for formulation $\mathbf{B}$, the shadow prices reflect the constraint shadow price (Lappi 1992). This could be described as for formulation $\mathbf{A}$, the shadow prices are related to a single periodic income (i.e., period $t$ to period 1); in this way, the shadow prices can be easily compared as they measure the marginal increase of period $t$ with a change in period 1 . In formulation B, the shadow prices are related to two periodic incomes (i.e., period $t$ to period $t-1$ ) and are not easily comparable, as they measure a marginal change dependent on the previous period. The shadow prices of the different formulations are directly related, as the variable shadow prices for each formulation are the same (to calculate variable shadow prices from constraint shadow prices readers are referred to Lappi (1992)).

\section{Conclusions}

Depending upon the risk preferences of the DM, the RP can find appropriate solutions which reflect the uncertainty of the data. The value of RP solution provides an improved solution for all risk preferences but is of more benefit for an individual who is very risk averse, rather than only mildly risk averse. For the specific case where even the slightest negative 
deviation is unwanted, the RP formulation will provide a very robust solution if $\lambda$ is set at a high value and even slight negative deviations will be unlikely. Additionally, through incorporating uncertainty into the optimization process, the RP will produce a more robust solution than the EV solution which ignores uncertainty. This makes intuitive sense, as more information is included in the decision-making process.

The problem formulation focused on ensuring even-flow of income over a small forest holding, incorporating a single source of error. The even-flow problem is only one potential application of stochastic programming in forest management planning. A variety of risk measurement tools (i.e., downside mean semideviation (Krzemienowski and Ogryczak 2005) or Conditional Value at Risk (Rockafellar and Uryasev 2000)) can be applied to the planning of future management actions on forest holdings. These risk measures need not be limited to economic criteria; risk measures could also be used to evaluate the capability of a plan to ensure the provision of specific ecosystem services or the viability of threatened/endangered species. Furthermore, to make this method more robust, additional sources of error can be incorporated directly to the scenario generation process. The impact of adding additional sources of errors should be studied in more depth.

Acknowledgments This study was supported by the Woodwisdom net project RegioPower.

\section{References}

Bertsimas D, Sim M (2004) The price of robustness. Oper Res 52:35-53 Birge JR, Louveaux F (2011) Introduction to stochastic programming. Springer

Diaz-Balteiro L, Romero C (2008) Making forestry decisions with multiple criteria: a review and an assessment. Forest Ecol Manag 255: 3222-3241

Diaz-Balteiro L, González-Pachón J, Romero C (2013) Goal programming in forest management: customising models for the decisionmaker's preferences. Scand J For Res 28:166-173

Duvemo K, Lämås T, Erikkson LO, Wikström P (2014) Introducing costplus-loss analysis into a hierarchical forestry planning environment. Ann Oper Res 219:415-431

Eid T (2000) Use of uncertain inventory data in forestry scenario models and consequential incorrect harvest decisions. Silva Fennica 34:89 100

Eyvindson K, Kangas A (2014) Stochastic goal programming in forest planning. Can J For Res 44:1274-1280. doi:10.1139/cjfr-2014-0170

Gregoire TG, Valentine HT (2008) Sampling strategies for natural resources and the environment. CRC Press, Boca Raton
Haara A, Korhonen K (2004) Kuvioittaisen arvioinnin luotettavuus. (reliability of compartmentwise inventory). Metsätieteen aikakauskirja 4:489-508 [in Finnish]

Hanewinkel M, Hummel S, Albrecht A (2011) Assessing natural hazards in forestry for risk management: a review. Eur J For Res 130:329 351

Hof JG, Pickens JB (1991) Chance-constrained and chance-maximizing mathematical programs in renewable resource management. For Sci 37:308-325

Islam MN, Kurttila M, Mehtätalo L, Pukkala T (2010) Inoptimality losses in forest management decisions caused by errors in an inventory based on airborne laser scanning and aerial photographs. Can J For Res 40:2427-2438

Kangas A, Hartikainen M, Miettinen K (2014) Simultaneous optimization of harvest schedule and measurement strategy. Scand J For Res 29:224-233. doi:10.1080/02827581.2013.823237

King AJ, Wallace SW (2012) Modeling with stochastic programming. Springer, New York

Knight FH (1921) Risk, uncertainty and profit. Hart, Schaffner and Marx, New York

Krzemienowski A, Ogryczak W (2005) On extending the LP computable risk measures to account downside risk. Comput Optim Appl 32: $133-160$

Lappi J (1992) JLP: A linear programming package for management planning. Research Papers 414, The Finnish Forest Research Institute, $134 \mathrm{pp}$

Leskinen P, Kangas J (2001) Modelling future timber price development by using expert judgments and time series analysis. Silva Fenn 35: 93-102

Mäkinen A, Kangas A, Mehtätalo L (2010) Correlations, distributions, and trends in forest inventory errors and their effects on forest planning. Can J For Res 40:1386-1396

Mulvey J, Vanderbei R, Zeinos S (1995) Robust optimization of largescale systems. Oper Res 43:264-281

Næsset E (2004) Accuracy of forest inventory using airborne laser scanning: evaluating the first Nordic full-scale operational project. Scand J For Res 19:554-557. doi:10.1080/02827580410019544

Noyan N (2012) Risk-averse two-stage stochastic programming with an application to disaster management. Comput Oper Res 39:541-559

Palma CD, Nelson JD (2009) A robust optimization approach protected harvest scheduling decisions against uncertainty. Can J For Res 39: $342-355$

Peltola, A. (Ed.) 2014 Finnish Statistical Yearbook of Forestry, Finnish Forest Research Institute http://www.metla.fi/metinfo/tilasto/ julkaisut/vsk/2014/index.html Accessed 5 May 2015

Piazza A, Pagnoncelli K (2014) The optimal harvesting problem under price uncertainty. Ann Oper Res 217:425-445

Pukkala T (2005) Metsikön tuottoarvon ennustemallit kivennäismaan männiköille, kuusikoille ja rauduskoivikoille (prediction models for productive value of pine, spruce and birch stands in mineral soils). Metsätieteen aikakauskirja 3:311-322 [in Finnish]

Rockafellar RT, Uryasev S (2000) Optimization of conditional value-atrisk. J Risk 2:21-42

Savage DW, Martell DL, Wotton BM (2011) Forest management strategies for dealing with fire-related uncertainty when managing two forest seral stages. Can J For Res 41:309-320 